\title{
Decreased microRNA-181a and -16 expression levels in the labial salivary glands of Sjögren syndrome patients
}

\author{
YUHUA WANG, GUOHUA ZHANG, LINGLING ZHANG, MIANSONG ZHAO and HONGDONG HUANG \\ Department of Rheumatology and Clinical Immunology, Beijing Shijitan Hospital, \\ Capital Medical University, Beijing 100038, P.R. China
}

Received January 23, 2017; Accepted July 20, 2017

DOI: $10.3892 /$ etm.2017.5407

\begin{abstract}
Sjögren syndrome (SS) is characterized by dysfunction of the exocrine glands, particularly the salivary and lacrimal glands. Thus, labial salivary gland biopsy is useful method for diagnosing SS. The aim of the present study was to investigate the microRNA (miRNA or miR) profile of labial salivary glands obtained from SS patients and to examine the correlation of miR-181a and -16 levels with the pathological grade in SS. miRNA expression in labial salivary gland tissues was profiled in 3 female patients with primary SS and 3 female patients with non-SS sicca syndrome using microarray analysis. In addition, a literature search and miRNA target gene prediction were performed to collect miRNAs involved in SS pathogenesis. Subsequent to integrating all database results, miR-181a and -16 were identified to be associated with the Ro/SS-associated antigen $\mathrm{A}$ and La/SS-associated antigen B during SS pathogenesis. Therefore, these miRNAs were selected for further verification in labial salivary gland tissues of 28 patients with SS and 18 non-SS sicca syndrome control individuals by quantitative reverse transcription-quantitative polymerase chain reaction. Compared with the control group, 76 miRNAs were upregulated and 51 were downregulated in the labial salivary gland of SS patients according to microarray results. In particular, miR-181a and -16 expression levels in the labial salivary gland of SS patients were decreased in comparison with those in the controls. Furthermore, the decreased expression levels of these miRNAs were associated with the labial salivary pathological focus scores. In conclusion, the present study examined the miRNA profiles in the labial salivary glands of SS patients and detected decreased miR-181a and -16 expression levels compared with the control individuals. Finally, the decreased levels of miR-181a and -16 were associated with the salivary gland pathological focus
\end{abstract}

Correspondence to: Dr Yuhua Wang, Department of Rheumatology and Clinical Immunology, Beijing Shijitan Hospital, Capital Medical University, 10 Tieyi Road, Beijing 100038, P.R. China

E-mail: wangyh@bjsjth.cn

Key words: Sjögren syndrome, labial salivary gland, microRNA-181a, microRNA-16 scores, suggesting that miR-181a and -16 may serve a role in the pathogenesis of SS.

\section{Introduction}

Sjögren syndrome (SS) is a chronic systemic inflammatory disease affecting the exocrine glands, particularly the salivary and lacrimal glands, resulting in keratoconjunctivitis sicca and xerostomia (1). The disease primarily affects middle-aged females. However, establishing the diagnosis of SS is often challenging, since the symptoms are nonspecific, while no single laboratory test allows for a definitive diagnosis and there are no disease-specific diagnostic criteria (2). Although the exact pathogenic mechanisms are not yet fully elucidated, immune system abnormalities are considered to be important in SS pathogenesis, including hypergammaglobulinemia, increased levels of autoantibodies against Ro/SS-associated antigen A (Ro/SSA) and La/SS-associated antigen B (La/SSB) (3), and exocrine gland histology characterized by focal lymphocyte infiltrates surrounding the glandular ducts $(4,5)$. However, it is unlikely that the pathogenesis of SS is solely immunologically mediated, and the contribution of nonimmune mechanisms should be considered. Therefore, the investigation of SS is appealing, particularly with respect to the role of numerous microRNAs (miRNAs or miR) involved in systemic autoimmune diseases, including their potential as diagnostic or prognostic biomarkers, as well as their roles in the pathogenesis of autoimmunity, inflammation and/or organ dysfunction.

miRNAs are highly conserved, small, noncoding, single-stranded RNA molecules that are able to regulate gene expression by binding to complementary sequences within the 3'-untranslated region of targeted mRNAs, leading to translational repression or degradation (6). miRNAs participate in various physiological and pathological processes, including the cellar metabolism, differentiation, proliferation and apoptosis. These molecules have been demonstrated to be potential diagnostic/prognostic biomarkers and/or therapeutic targets in various diseases, including hepatocellular carcinoma, multiple myeloma and cardiac diseases (7). In addition, miRNAs participate in the regulation of innate and adaptive immune responses (8). At present, miRNAs have attracted increasing attention due to their roles in the pathogenesis of rheumatic diseases. For instance, dysregulation of miRNAs has been 
reported to be associated with the disease activity and major organ involvement in patients with systemic lupus erythematosus and rheumatoid arthritis (RA) (9). However, only a limited number of studies have focused on understanding the miRNA function in SS. It has been reported that miR-146a, -155 and -181a were overexpressed in peripheral blood mononuclear cells (PBMCs) collected from SS patients (7,10-13), while miR-17 to -92 were downregulated in the salivary gland tissues (12). Although regulated expression of certain miRNAs have been demonstrated in the salivary gland tissues of SS patients, a comprehensive description of their contribution to the pathological grade of SS remains incomplete.

In the current study, the miRNA profile of labial salivary glands from SS patients and control subjects were investigated and the correlation between miRNAs and SS pathological grade was examined.

\section{Materials and methods}

Patients. SS patients $(n=28)$ were recruited from the Department of Rheumatology of Beijing Shijitan Hospital (Beijing, China) between August 2008 and August 2013. The inclusion criteria for SS patients included the following: i) Fulfillment of all revised criteria for SS diagnosis proposed by the American-European Consensus (AEC) Group (14); ii) absence of any other rheumatic or underlying disease; and iii) no previous treatment with glucocorticoid or immunosuppressive agents. Furthermore, 18 age- and sex-matched non-SS sicca syndrome patients recruited from Beijing Shijitan Hospital were used as controls. Non-SS sicca syndrome was defined as the presence of xerostomia and xerophthalmia in patients with negative non-organ-specific autoantibodies and a negative minor salivary gland biopsy according to the Chisolm and Mason scoring system (15). Detailed information on the SS and control patients is provided in Table I. The current study was approved by the Ethics Committee of the Capital Medical University (Beijing, China). Informed written consent was obtained from all patients participating in the present study.

Salivary gland tissue specimens. As part of the diagnostic workup, all SS and control patients underwent salivary gland biopsy, according to the AEC Group guidelines. Briefly, a local anesthetic was injected into the lower lip, followed by performing a small incision to the right or left of the midline of the lip. A total of 5 or 6 minor salivary gland lobules were harvested, and all specimens had a glandular area of $\geq 4 \mathrm{~mm}^{2}$. Following harvest, the majority of specimens were stored at $-80^{\circ} \mathrm{C}$ for miRNA expression profiling, while the remaining samples were delivered directly to the pathology department for histopathological evaluation. Evaluation of biopsy samples from SS and control patients was performed independently by two oral pathologists. Specimens from SS patients demonstrated characteristic features of SS, including a salivary gland pathological focus (SGPF) score of $\geq 1$ and presence of lymphoepithelial lesions (16), whereas specimens obtained from the control subjects did not present such features. Subsequently, SS patients were divided into two groups based on their SGPF scores, as follows: Scores of $<3(n=16)$ and scores of $\geq 3(n=12)(17)$.
RNA isolation and microarray analysis. Total RNA was harvested from the labial salivary gland tissues from all SS and control subjects using TRIzol reagent (Invitrogen; Thermo Fisher Scientific, Inc., Waltham, MA, USA) and miRNeasy mini kit (Qiagen, Hilden, Germany) according to the manufacturer's protocol, in order to efficiently recover all RNA species, including miRNAs. The total RNA concentration was then measured using a NanoDrop 1000 device (Thermo Fisher Scientific, Inc.) at an optical density of 260/280 nm, and then samples were labeled using a miRCURY ${ }^{\mathrm{TM}} \mathrm{Hy} 3^{\mathrm{TM}} / \mathrm{Hy}^{\mathrm{TM}}$ miRNA Power Labeling kit (Exiqon; Qiagen) and hybridized on a miRCURY ${ }^{\mathrm{TM}}$ LNA miRNA Array (version 18.0; Exiqon; Qiagen). Subsequent to washing, arrays were scanned using an Axon GenePix 4000B microarray scanner (Molecular Devices, LLC, Sunnyvale, CA, USA).

Microarray expression profiling of miRNAs in the labial salivary gland tissues was performed on 3 randomly selected female patients with SS, and 3 randomly selected age- and sex-matched patients with non-SS sicca syndrome. This was performed using a seventh generation, single-color miR CURY LNA $^{\mathrm{TM}}$ miRNA Array that contained 3,100 capture probes, covering all the human, mouse and rat miRNAs annotated in the miRBase database (version 18.0; mirbase.org), as well as all the viral miRNAs associated with these species. In addition, this array contained capture probes for 25 proprietary miRPlus ${ }^{\mathrm{TM}}$ human miRNAs (Exiqon; Qiagen) that were not found in the miRBase database. Gene expression was normalized using the median normalization method, as follows: Normalized data $=($ foreground-background $) /$ median expression, where median expression is the $50 \%$ quantile of miRNA intensity $>50$ in all samples subsequent to background correction. The threshold value used to define the significant upregulation or downregulation of miRNAs in patients with SS compared with the controls was a fold-change in the expression level of $>1.5$ and a P-value of $<0.05$ according to Student's t-test.

The Ro/SSA and La/SSB intracellular ribonucleoproteins are the major targets of autoimmune humoral responses in $\mathrm{SS}$ (18). A literature review found that there were $30 \mathrm{miRNAs}$, including miR-181a, miR-16, target Ro/SSA (Ro52/E3 ubiquitin-protein ligase TRIM21 or Ro60/60 kDa SS-A/Ro ribonucleoprotein) and La/SSB mRNAs (19). miRNA target prediction programs, TargetScan (targetscan.org, version 7.1) and MicroRNA (microrna.org, 2010 release), were used to double-check these miRNAs target genes and validate their functions. Subsequently, the differential expression of miR-181a and miR-16 in the tissues of patients with SS were confirmed by reverse transcription-quantitative polymerase chain reaction (RT-qPCR) analysis.

miRNA validation by RT-qPCR analysis. Subsequent to integrating all database results, miR-181a and -16 were observed to be associated with La/SSA and Ro/SSB during SS pathogenesis. Therefore, these two miRNAs were verified in the labial salivary gland samples of all 28 patients with SS and 18 control patients. Total RNA was reverse transcribed into cDNA using a Taqman miRNA Reverse Transcription kit (Applied Biosystems; Thermo Fisher Scientific, Inc.). qPCR was then conducted using TaqMan ${ }^{\mathrm{TM}}$ Fast Advanced Master Mix (Thermo Fisher Scientific, Inc.) according to the manufacturer's protocol. Briefly, a $20 \mu \mathrm{l}$ PCR reaction included $2 \mu \mathrm{l}$ RT product, $1 \mu \mathrm{l} \mathrm{TaqMan}{ }^{\mathrm{TM}}$ 
Table I. Demographic data and clinical characteristics of patients with SS and controls.

\begin{tabular}{lcc}
\hline Variable & SS (n=28) & Controls (n=18) \\
\hline $\begin{array}{l}\text { Demographics } \\
\text { Male/female }\end{array}$ & $1 / 27$ & $1 / 17$ \\
Age, years & $50.14 \pm 2.65$ & $54.23 \pm 3.21$ \\
Clinical manifestations & & \\
Dry eye, no. +/- & $22 / 6$ & $11 / 7$ \\
Dry mouth, no. +/- & $22 / 6$ & $14 / 4$ \\
Organ involvement, no. +/- & $12 / 16$ & - \\
Laboratory tests & & \\
ANA positivity, no. +/- & $18 / 10$ & - \\
Anti-Ro(SSA), no. +/- & $14 / 14$ & - \\
Anti-La(SSB), no. +/- & $8 / 20$ & - \\
RF, no. +/- & $12 / 16$ & \\
Elevated ESR, no. +/- & $18 / 10$ & - \\
\hline
\end{tabular}

Data are presented as the mean \pm standard error or as a ratio. miR, microRNA; ANA, anti-nuclear antibody; SS, Sjögren syndrome; Ro/SSA, Ro/SS-associated antigen A; La/SSB, La/SS-associated antigen B; RF, rheumatoid factor; ESR, erythrocyte sedimentation rate; no., number; +, with; -, without.

Gene Expression Assay (20X), $10 \mu \mathrm{l}$ TaqMan $^{\mathrm{TM}}$ Fast Advanced Master Mix (10X), $7 \mu$ nuclease-free water. The primers were synthesized by the Sangon Biotech Co., Ltd. (Shanghai, China) and were as follows: miR-181a, 5'-GGGAACATTCAACGC TGTCG-3' (forward) and 5'-GTGCGTGTCGTGGAGTCG-3' (reverse); miR-16, 5'-GGGTAGCAGCACGTAAATA-3' (forward) and 5'-CAGTGCGTGTCGTGGAGT-3' (reverse); U6, 5'-GCTTCGGCAGCACATATACTAAAAT-3' (forward) and 5'-CGCTTCACGAATTTGCGTGTCAT-3' (reverse). The reactions were carried out at $95^{\circ} \mathrm{C}$ for $10 \mathrm{~min}$, followed by 40 cycles of $95^{\circ} \mathrm{C}$ for $15 \mathrm{sec}$ and $60^{\circ} \mathrm{C}$ for $1 \mathrm{~min}$. Relative gene expression was calculated by the $2^{-\Delta \Delta C q}$ method (20) and snRNA U6 was used as an internal control.

Statistical analysis. The results of the microarray analysis of miRNA expression between the SS and control patients were compared using an unpaired Student's t-test, where a $\mathrm{P}<0.05$ and fold-change of $>2.0$ were considered statistically significant. Statistical analysis was performed using SPSS version 19.0 software (IBM Corp., Armonk, NY, USA). A Mann-Whitney test was used to assess the differences between controls and patients with SS, and a $\mathrm{P}<0.05$ was considered as an indicator of statistically significant differences.

\section{Results}

Differentially expressed miRNAs in labial salivary gland tissues. Since altered expression levels of several miRNAs have been reported in the PBMCs of patients with SS (21). The present study further investigated the dysregulation of miRNAs in the labial salivary gland tissue. In order to identify miRNAs that may correlate with SS, microarray analysis was used to investigate the miRNA expression profiles in labial salivary gland tissues from 3 patients with SS and 3 patients with non-SS sicca syndrome. The results revealed a total of 127 differentially expressed miRNAs in SS patients when compared with their levels in the control patients, including 76 upregulated and 51 downregulated miRNAs (Fig. 1).

miR-181a and -16 expression levels in labial salivary gland tissues. In order to identify miRNAs potentially associated with SS, a literature search and miRNA target prediction was performed using TargetScan and MicroRNA.org. Through this analysis two miRNAs, miR-181a and -16, were identified to be associated with Ro/SSA and La/SSB in patients with SS. miR-181a and -16 expression levels in 28 SS and 18 control labial salivary gland tissues were detected by RT-qPCR. As shown in Fig. 2, the miR-181a and -16 expression levels were significantly downregulated in patients with SS compared with the controls $(\mathrm{P}<0.01)$, suggesting that miR-181a and -16 may serve a role in the pathogenesis of SS.

Correlation of miR-181a and -16 expression levels with SGPF scores. To further characterize the relevance of miR-181a and -16 expression levels in SS labial salivary glands, statistical analyses were performed on SS patients grouped according to their SGPF score. It was observed that the levels of miR-181a and -16 were increased in patients with SS who possessed high SGPF scores of $\geq 3$ (high-grade inflammation) when compared with those in patients exhibiting low SGPF scores of $<3$ (low-grade inflammation; Fig. 3). This indicates that miR-181a and -16 may be involved in the pathogenesis of SS.

\section{Discussion}

SS is a chronic, inflammatory, autoimmune disease characterized by lymphocytic infiltration of the exocrine glands, particularly of the salivary and lacrimal glands, leading to destruction of their functional components (1). However, SS diagnostic criteria, including the AECG serological or histopathological criteria in conjunction with oral and ocular dryness, are frequently inconsistently applied and cannot optimally differentiate patients with SS from patients with non-SS sicca or address extraglandular manifestations. As a result, the diagnosis of SS may be delayed by 6-10 years after the disease onset, while recent attempts to address these issues by novel diagnostic criteria for early SS that were reported in 2015 require further large-scale validation (22). According to the mentioned SS criteria, labial salivary gland biopsy occupies a very important position in the diagnosis of SS $(14,23)$, although this is an invasive procedure. Recently, an increasing number of studies revealed that miRNAs in PBMCs, sera and saliva contribute to the pathogenesis of SS and may represent a novel class of diagnostic biomarkers for this disease $(8,10,11,21)$. However, studies investigating the miRNA profiles in the salivary gland tissue of SS patients are currently lacking. In the current study, microarray analysis demonstrated the upregulation of 76 miRNAs and downregulation of 51 miRNAs in the labial salivary gland tissues of SS patients relative to the controls. miR-181a and -16 were observed to be associated with and to regulate Ro/SSA and La/SSB during SS pathogenesis. Therefore, their expression was further verified in labial 


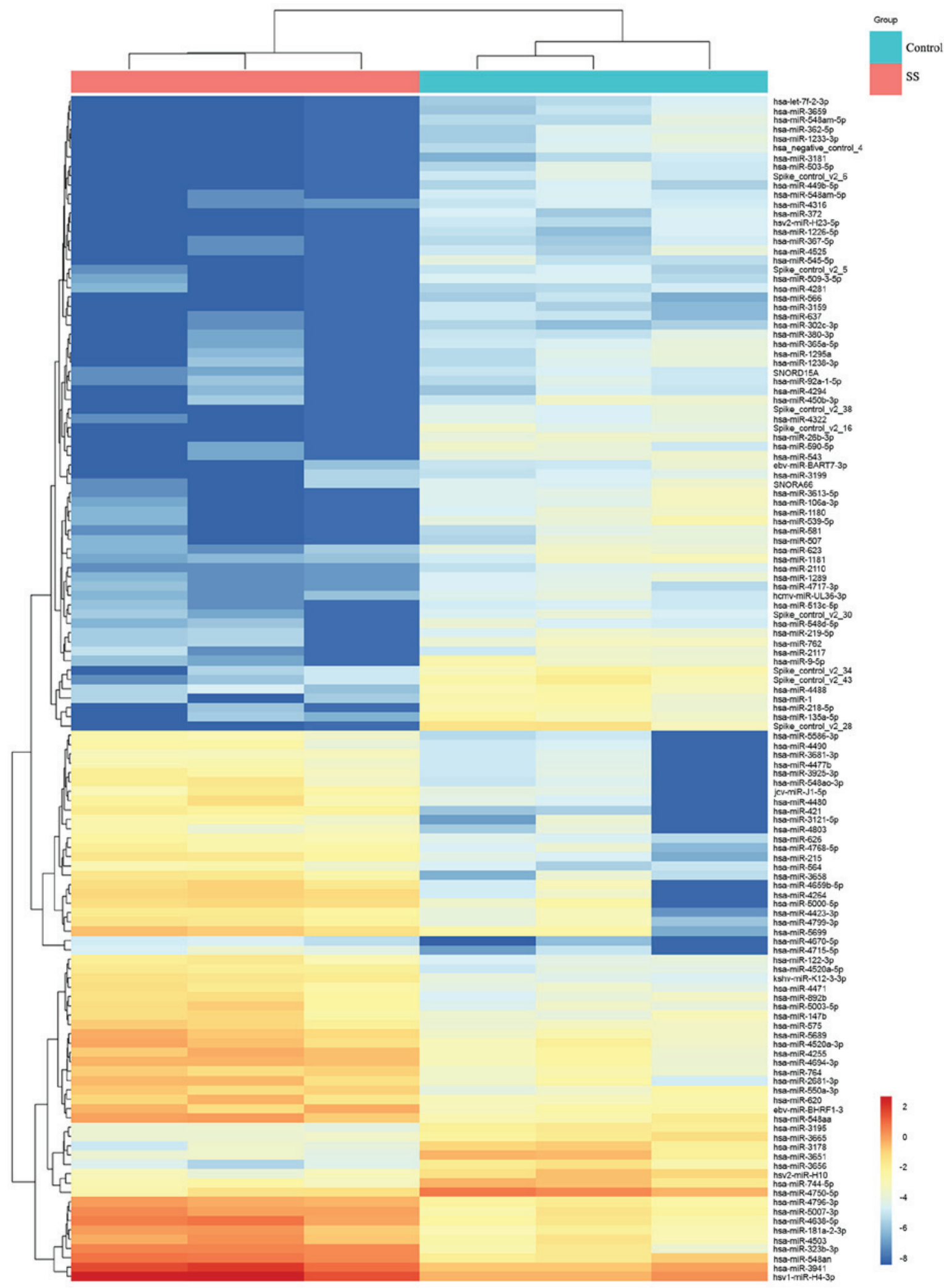

Figure 1. Microarray analysis of labial salivary gland tissues from SS and control patients. The hierarchically clustered heat map illustrates alterations in miRNA expression profiles between the patients with primary SS and patients with non-SS sicca syndrome (control). The cluster of significantly expressed miRNA was identified using a Student's t-test $(\mathrm{P}<0.05)$. Red and green sections represent increases and decreases in miRNA expression, respectively. miR, microRNA; SS, Sjögren syndrome.

salivary gland tissues from 28 patients with SS and 18 controls included in the present study. The results revealed that the expression levels of miR-181a and -16 were decreased in SS patients compared with those in control subjects. Furthermore,
miR-181a and -16 expression levels were associated with the labial SGPF score.

miR-181a has been reported to be involved in hematological malignancies (24). For instance, miR-181a was 
A

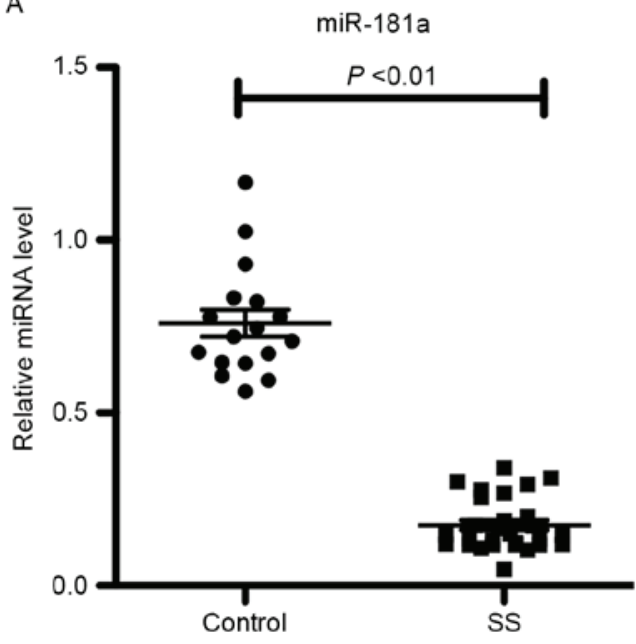

B

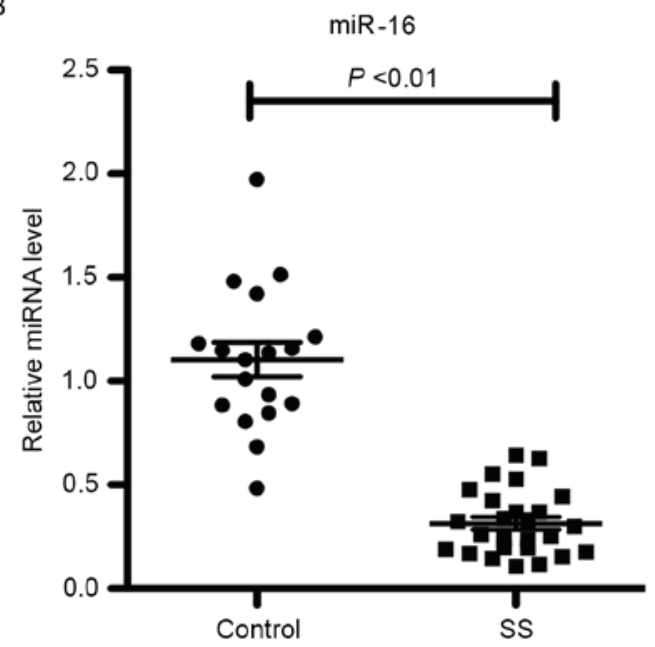

Figure 2. miR-181a and -16 expression levels in labial salivary gland tissues obtained from SS and control patients. Detection of (A) miR-181a and (B) miR-16 expression levels was conducted by reverse transcription-quantitative polymerase chain reaction. miR-181a and -16 levels were decreased in SS patients as compared with the control patients. miR/miRNA, microRNA; SS, Sjögren syndrome.

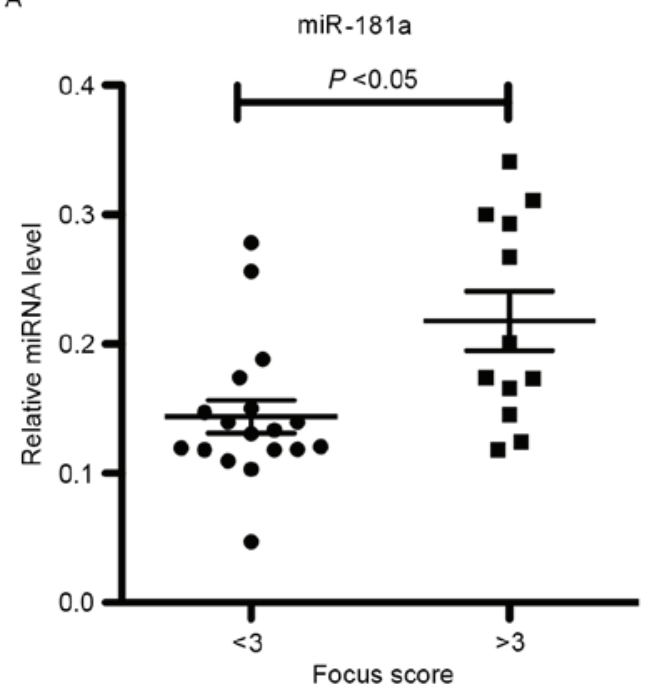

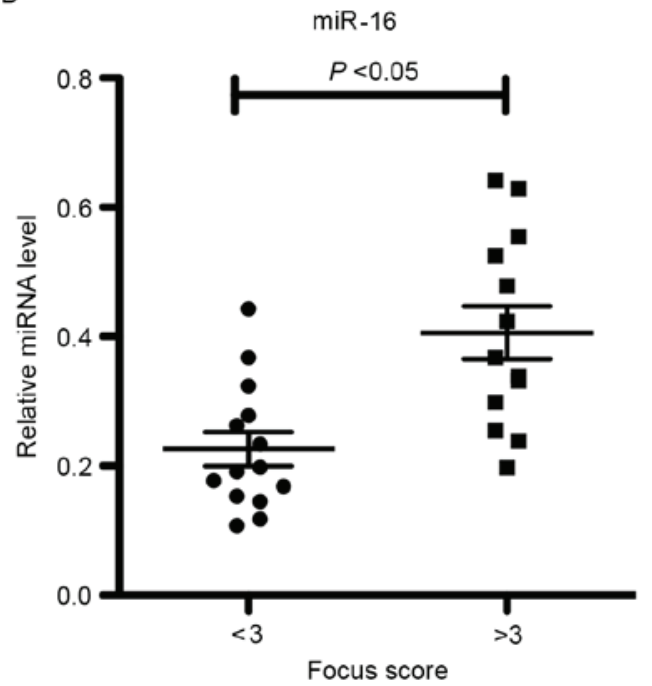

Figure 3. Relative expression levels of (A) miR-181a and (B) miR-16 in the labial salivary gland tissues obtained from SS patients. Patients with SS were divided into two groups based on their SGPF score ( $<3,16$ patients; $\geq 3,12$ patients). The expression levels of miR-181a and miR-16 were increased in patients with high SGPF scores compared with patients with low SGPF scores. miR/miRNA, microRNA; SGPF, salivary gland pathological focus.

overexpressed in T-/B-cell leukemia/lymphoma, while miR-181a-deficient mice demonstrated severe defects in T-cell development (24-26). This indicates that miR-181a may be involved in the pathogenesis of lymphocytic diseases. In the present study, miR-181a expression was significantly downregulated in the labial salivary gland tissues of patients with SS in comparison with the controls. In contrast to the present observations, Peng et al (27) reported that miR-181a expression was elevated in PBMCs of Chinese patients with SS, and there was a positive correlation between miR-181a levels and antinuclear antibody titer. The overexpression of miR-181a contributed to the B-cell maturation rather than that of T-cells in patients with SS. Although this observation is inconsistent with the results of the present study, it should be noted that the miRNA profiles in different tissues were examined in the two studies. Since the function and effect of miRNA is dependent on the tissue in numerous cases, identifying the cell types that present miRNA dysregulation is imperative to the study of their role in disease (28). Furthermore, miR-181a expression has been demonstrated to increase the number of B-lymphoid cells, with a concurrent decrease in T-lymphoid cells (28). Peng et al (27) reported that individual measurement of the miR-181a level in B- and T-cells isolated from PBMCs indicated that the B-cell population contributed to the elevation in miR-181a levels, which may be involved in the increased miR-181a levels in PBMCs and decreased levels in labial salivary gland tissues. Another study by Li et al (29) reported that increasing the miR-181a expression in mature T-cells augments their sensitivity to peptide antigens, while inhibiting the miR-181a expression in immature T-cells reduces their sensitivity and impairs the positive and negative selection. T-cell sensitivity to autoantigens is quantitatively 
regulated by managing the expression of miR-181a in order to enable mature T-cells to recognize antagonists (inhibitory peptide antigens) as agonists. Li et al (29) also revealed a correlation between higher miR-181a levels and greater sensitivity in immature T-cells, which may explain the elevated miR-181a levels in the salivary glands of SS patients with high-grade inflammation.

Numerous studies have indicated that miR-16 family members regulate cell cycle-associated genes, as well as promote tumorigenesis and metastasis $(30,31)$. Hence, miR-16 family members have been considered as potential biomarkers in cancer diagnosis (32). Certain studies have recently reported altered miR-16 expression in rheumatoid disease. For instance, Pauley et al (33) identified miR-16 overexpression in the PBMCs of RA patients with active disease as compared with RA patients with low disease activity or healthy control patients. Another study demonstrated that higher levels of miR-16 in the sera of treatment naïve patients with early-stage RA correlated with attenuation of the disease (34). Thus, monitoring miR-16 levels may be a useful tool for predicting the disease outcomes in patients with early-stage RA. In the current study, reduced miR-16 expression was observed in the labial salivary glands of patients with SS compared with that in patients with non-SS sicca syndrome (controls), however, the patients with SS who exhibited high SGPF scores in salivary glands had elevated level of miR-16.

To the best of our knowledge, the present study is the first to report decreased expression levels of miR-181a and -16 in the labial salivary gland of SS patients; however, this study has several limitations. Firstly, the current study only verified the expression levels of miR-181a and -16 in labial salivary gland tissues. In the future, we intend to investigate the expression of miRNAs in PBMCs and labial salivary gland tissues simultaneously in a large cohort of patients. In addition, the precise mechanism through which miR-181a and -16 affect the focal lymphocytic infiltration should be further clarified.

In conclusion, the present investigation of miRNA profiles in the labial salivary gland of SS and control patients demonstrated reduced expression levels of miR-181a and -16 in conjunction with SS. Furthermore, these two miRNAs were increased in patients with SS who possessed high SGPF scores, suggesting that miR-181a and -16 may serve a role in the pathogenesis of SS.

\section{Acknowledgements}

The present study was supported by the Beijing National Science Foundation (grant no. 7123220). The authors would like to thank all the patients enrolled into the present study, and Dr. Xiaoyang Jin (Institute of Biophysics, Chinese Academy of Science, Beijing, China) for his special contribution to the investigation.

\section{References}

1. Tincani A, Andreoli L, Cavazzana I, Doria A, Favero M, Fenini MG, Franceschini F, Lojacono A, Nascimbeni G, Santoro A, et al: Novel aspects of Sjögren's syndrome in 2012. BMC Med 11: 93, 2013.

2. Cotrim AP and Alevizos I: Human and viral microRNA expression in Sjögren syndrome. J Rheumatol 41: 2102-2103, 2014.
3. Mackay F, Groom JR and Tangye SG: An important role for B-cell activation factor and B cells in the pathogenesis of Sjögren's syndrome. Curr Opin Rheumatol 19: 406-413, 2007.

4. Greenspan JS, Daniels TE, Talal N and Sylvester RA: The histopathology of Sjögren's syndrome in labial salivary gland biopsies. Oral Surg Oral Med Oral Pathol 37: 217-229, 1974.

5. Fox PC: Autoimmune diseases and Sjogren's syndrome: An autoimmune exocrinopathy. Ann N Y Acad Sci 1098: 15-21, 2007.

6. Chua JH, Armugam A and Jeyaseelan K: MicroRNAs: Biogenesis, function and applications. Curr Opin Mol Ther 11: 189-199, 2009.

7. Bartel DP: MicroRNAs: Genomics, biogenesis, mechanism, and function. Cell 116: 281-297, 2004.

8. Shi H, Zheng LY, Zhang P and Yu CQ: miR-146a and miR-155 expression in PBMCs from patients with Sjögren's syndrome. J Oral Pathol Med 43: 792-797, 2014.

9. Carlsen AL, Schetter AJ, Nielsen CT, Lood C, Knudsen S, Voss A, Harris CC,Hellmark T, Segelmark M, Jacobsen S, et al: Circulating microRNA expression profiles associated with systemic lupus erythematosus. Arthritis Rheum 65: 1324-1334, 2013.

10. Tandon M, Gallo A, Jang SI, Illei GG and Alevizos I: Deep sequencing of short RNAs reveals novel microRNAs in minor salivary glands of patients with Sjögren's syndrome. Oral Dis 18: 127-131, 2012.

11. Gallo A, Tandon M, Illei G and Alevizos I: Discovery and validation of novel microRNAs in Sjögren's syndrome salivary glands. Clin Exp Rheumatol 32: 761-762, 2014.

12. Alevizos I, Alexander S, Turner RJ and Illei GG: MicroRNA expression profiles as biomarkers of minor salivary gland inflammation and dysfunction in Sjögren's syndrome. Arthritis Rheum 63: 535-544, 2011

13. Pauley KM, Stewart CM, Gauna AE, Dupre LC, Kuklani R, Chan AL, Pauley BA, Reeves WH, Chan EK and Cha S: Altered miR-146a expression in Sjögren's syndrome and its functional role in innate immunity. Eur J Immunol 41: 2029-2039, 2011.

14. Vitali C, Bombardieri S, Jonsson R, Moutsopoulos HM, Alexander EL, Carsons SE, Daniels TE, Fox PC, Fox RI, Kassan SS, et al: Classification criteria for Sjögren's syndrome: A revised version of the European criteria proposed by the American-European Consensus Group. Ann Rheum Dis 61: 554-558, 2002.

15. Chisholm DM and Mason DK: Labial salivary gland biopsy in Sjögren's disease. J Clin Pathol 21: 656-660, 1968.

16. Baldini C, Giusti L, Ciregia F, Da Valle Y, Giacomelli C, Donadio E, Sernissi F, Bazzichi L, Giannaccini G, Bombardieri S and Lucacchini A: Proteomic analysis of saliva: A unique tool to distinguish primary Sjögren's syndrome from secondary Sjögren's syndrome and other sicca syndromes. Arthritis Res Ther 13: R194, 2011.

17. Daniels TE, Cox D, ShiboskiCH, Schiødt M, Wu A,LanfranchiH, Umehara H, Zhao Y, Challacombe S, Lam MY, et al: Associations between salivary gland histopathologic diagnoses and phenotypic features of Sjögren's syndrome among 1,726 registry participants. Arthritis Rheum 63: 2021-2030, 2011.

18. Gourzi VC, Kapsogeorgou EK, Kyriakidis NC and Tzioufas AG: Study of microRNAs (miRNAs) that are predicted to target the autoantigens Ro/SSA and La/SSB in primary Sjögren's Syndrome. Clin Exp Immunol 182: 14-22, 2015.

19. Kapsogeorgou EK, Gourzi VC, Manoussakis MN, Moutsopoulos HM and Tzioufas AG: Cellular microRNAs (miRNAs) and Sjögren's syndrome: Candidate regulators of autoimmune response and autoantigen expression. J Autoimmun 37; 129-135, 2011.

20. Livak KJ and Schmittgen TD: Analysis of relative gene expression data using real-time quantitative PCR and the 2(-Delta Delta C(T)) method. Methods 25: 402-408, 2001

21. Chen JQ, Papp G, Szodoray P and Zeher M: The role of microRNAs in the pathogenesis of autoimmune diseases. Autoimmun Rev 15: 1171-1180, 2016.

22. Brito-Zerón P, Theander E, Baldini C, Seror R, Retamozo S, Quartuccio L, Bootsma H, Bowman SJ, Dorner T, Gottenberg JE, et al: Early diagnosis of primary Sjögren's syndrome: EULAR-SS task force clinical recommendations. Expert Rev Clin Immunol 12: 137-156, 2016.

23. Shiboski SC, Shiboski CH, Criswell L, Baer A, Challacombe S, Lanfranchi H, Schiødt M, Umehara H, Vivino F, Zhao Y, et al: American College of Rheumatology classification criteria for Sjögren's syndrome: A data-driven, expert consensus approach in the Sjögren's International Collaborative Clinical Alliance cohort. Arthritis Care Res (Hoboken) 64: 475-487, 2012. 
24. Bi C and Chng WJ: MicroRNA: Important player in the pathobiology of multiple myeloma. BioMed Res Int 2014: 521586, 2014.

25. Chen CZ, Li L, Lodish HF and Bartel DP: MicroRNAs modulate hematopoietic lineage differentiation. Science 303: 83-86, 2004.

26. Henao-Mejia J, Williams A, Goff LA, Staron M, Licona-Limón P, Kaech SM, Nakayama M, Rinn JL and Flavell RA: The microRNA miR-181 is a critical cellular metabolic rheostat essential for NKT cell ontogenesis and lymphocyte development and homeostasis. Immunity 38: 984-997, 2013.

27. Peng L, MaW, Yi F, Yang YJ,Lin W, Chen H, Zhang X, Zhang LH, Zhang $F$ and Du Q: MicroRNA profiling in Chinese patients with primary Sjögren syndrome reveals elevated miRNA-181a in peripheral blood mononuclear cells. J Rheumatol 41: 2208-2213, 2014.

28. Alevizos I and Illei GG: MicroRNAs in Sjögren's syndrome as a prototypic autoimmune disease. Autoimmun Rev 9: 618-621, 2010.

29. Li QJ, Chau J, Ebert PJ, Sylvester G, Min H, Liu G, Braich R, Manoharan M, Soutschek J, Skare P, et al: miR-181a is an intrinsic modulator of T cell sensitivity and selection. Cell 129: 147-161, 2007
30. Aqeilan RI, Calin GA and Croce CM: miR-15a and miR-16-1 in cancer: Discovery, function and future perspectives. Cell Death Differ 17: 215-220, 2010.

31. Tung YT, Huang PW, Chou YC, Lai CW, Wang HP, Ho HC, Yen CC, Tu CY, Tsai TC, Yeh DC, et al: Lung tumorigenesis induced by human vascular endothelial growth factor (hVEGF)-A165 overexpression in transgenic mice and amelioration of tumor formation by miR-16. Oncotarget 6: 10222-10238, 2015.

32. Cui J: MiR-16 family as potential diagnostic biomarkers for cancer: A systematic review and meta-analysis. Int J Clin Exp Med 8: 1703-1714, 2015.

33. Pauley KM, Satoh M, Chan AL, Bubb MR, Reeves WH and Chan EK: Upregulated miR-146a expression in peripheral blood mononuclear cells from rheumatoid arthritis patients. Arthritis Res Ther 10: R101, 2008.

34. Filková M, Aradi B, Senolt L, Ospelt C, Vettori S, Mann H, Filer A, Raza K, Buckley CD, Snow M, et al: Association of circulating miR-223 and miR-16 with disease activity in patients with early rheumatoid arthritis. Ann Rheum Dis 73: 1898-1904, 2014. 